\title{
User needs and preferences on AAL systems that support older adults and their carers
}

\author{
Amedeo Cesta ${ }^{\mathrm{a},{ }^{*}}$, Gabriella Cortellessa ${ }^{\mathrm{a}}$, Francesca Fracasso ${ }^{\mathrm{a}}$, Andrea Orlandini ${ }^{\mathrm{a}}$ and Marcello Turno $^{\mathrm{b}}$ \\ ${ }^{a}$ Istituto di Scienze e Tecnologie della Cognizione, CNR, Via San Martino della Battaglia, 44, Rome, Italy \\ E-mails: amedeo.cesta@istc.cnr.it, gabriella.cortellessa@istc.cnr.it,francesca.fracasso@istc.cnr.it, \\ andrea.orlandini@istc.cnr.it \\ ${ }^{\mathrm{b}}$ Azienda Sanitaria Locale, ASL Roma A, Via Monte Rocchetta, 14, Rome, Italy \\ E-mail: mf_turno@iol.it
}

\begin{abstract}
This article describes a systematic investigation on the expectations of potential users of Ambient Assisted Living (AAL) technologies. More specifically it describes the efforts made to elicit users expectations for services of an AAL system that aims to both monitor older people fostering their independent living and preventing dangerous situations and also offers a valid help to their caregivers. The specific target of our work is older adults, with no critical pathological conditions, who wish to stay home as long as possible. The work proposes a plan for a rigorous systematization of the users needs and their validation based on a combination of qualitative and quantitative research techniques involving both the primary users (older users at home) and the secondary users (formal and informal caregivers) who constitute the network of persons around them. Four main areas relevant to the realization of personalized services have been identified that could help to support independent living and improve Quality of Life (QoL) which include the monitoring of Social Interaction, Physiological Data, Daily Activities and Environmental Data. The presented results are also consistent with the global theoretical framework of International Classification of the Functioning, Disability and Health (ICF) which highlights the main factors contributions to independent living. This study also investigates the perceived usefulness and acceptance of specific services (considering both primary and secondary users perspective) that have been listed inside each category with the aim to indicate a clear priority level and preferences of users. The article concludes by providing insights on practical implications for the systems development, presenting concrete examples of possible use cases relevant for the AAL domain linked to the elicited user requirements.
\end{abstract}

Keywords: User needs, ambient assisted living, user centered design, older people, personalized AAL services, prevention, independent living

\section{Introduction}

Life expectation is nowadays increasing [18] and consequently the number of people who will need support to cope with age-related impairments and to maintain a valuable Quality of Life (QoL) will also increase. One of the first wishes of this population is to be able to live autonomously as long as possible, and to age well. Notably, independent living is also known to reduce the cost to society of supporting people who have lost some autonomy [20] and there is consid-

${ }^{*}$ Corresponding author. E-mail: amedeo.cesta@ istc.cnr.it. erable evidence that health promoting behaviours of older people offers the potential for improving health status and Quality of Life also reducing the cost of health care $[3,23,26,33]$. Quality of Life has been defined as "an individual's perceptions of their position in life in the context of the culture and value system in which they live and in relation to their goals, expectations, standards and concern" [42, p. 1570]. Most researchers consider it to be a multidimensional concept encompassing health, functional status, social, as well as other aspects of an individual life $[6,36,38]$.

Advanced age is one of the most important factors for frailty, dependency, and level of received care 
$[2,14,25,27,29]$. In this respect, it becomes important to promote and foster a good QoL in such kind of persons, that also means to cope with age-related health impairments and decrease of independence. A correlated concept is the notion of Aging-In-Place [30] that promotes the idea of allowing seniors to keep control of their environment and activities to improve their autonomy, health, well-being and their feeling of dignity. The adoption of new technologies to preserve the independence of older people in the domestic environment is becoming increasingly important in the context of this demographic change and the relevance of this new technology for daily activities of self care [11] within the smart home domain is also increasingly discussed.

Initiatives to leverage technology as a key element to support ageing are indeed manifold, most of which fall under the Ambient Assisted Living (AAL) initiative funded by the European Commission. The purpose of this program is the development and use of new technologies to enable older users and disabled to live comfortably at home, improving their autonomy, facilitating daily activities, ensuring good conditions of safety, supporting prevention or monitoring and treating sick people. The widespread application of AAL technique could avoid, in many cases, the admission to hospitals or nursing homes, allowing a better QoL and savings costs for the community.

This work reports an effort performed in Italy to elicit, in a structured way, user expectations for an AAL system devoted to older adults (not affected by a critical pathologic condition) to support their independent living. The analysis is based on the combination of qualitative and quantitative methods involving representative users (ecological context). The specific effort derived from the quantitative approach allowed validating our results. Additionally the statistically relevant number of participants provides a clear indication of priorities for the synthesis of AAL services based on acceptance and perceived usefulness of both primary and secondary users. Detailed priorities are provided for specific services belonging to four different categories. The conclusions derived from this study can be used as an indication of the main wishes of older adults with respect to technology that is intended as a means to support their independent living and is also influenced by the perspective of their caregivers.

The paper is organized as follows: Section 2 contains an overview of related works on the investigations of users expectations from AAL technology; Section 3 describes the main concepts and ingredients that inspired the study and that emerged from our experi- ence, the GIRAFFPLUS project idea; Section 4 presents the core contribution of this paper, describing both the method used to elicit user expectations and the results of the study with specific emphasis on the assigned priorities; Section 5 discusses the results and their implications for the development of AAL technologies and illustrates some system scenarios that complete the analysis; Section 6 discusses some limitations of the current study while Section 7 concludes the paper.

\section{Related works}

\subsection{Smart homes, AmI and AAL: Users involvement}

A study by Eggen and colleagues [17] tried to investigate what "home" actually means for people, ultimately aiming at understanding how people would like to live in their "Dream Home", the "Home of the Future" highlighting some interesting requirements in terms of trust, usability and the possibility to control the smart devices. The paper is an example of codesign experience that aimed at eliciting needs of families for future homes using a qualitative approach. The participants were not older users who inevitably have specific features and limitation in their use and interaction with technologies. In the same direction, a survey about the users' expectations with respect to intelligent homes [5] highlights some of the main evident wishes of end users about next-generation homes that are mainly related to comfort and household tasks. Both these studies represent an attempt to overcome the discrepancy between current intelligent solutions and users expectancies, with the main aim to raise awareness within the Ambient Intelligence (AmI) community of the need to establish more effective communication with users in real-world deployment situations fostering the "user in the loop" approach. Moving from the AmI concept to the AAL domain, the general goal of AAL solutions is to apply AmI concepts and technologies to enable people with specific needs (e.g., the older users) to live longer in their natural environment. This inevitably poses additional challenged to developers who need to take into account the particular needs of the different users.

A recent review on AAL technologies, products and services [37] promotes a user-centric development of technology with a strong involvement of end users specifically for usability and accessibility issues pointing out the lack of proper attention to the topic. The review analyzes a number of papers related to the AAL 
research areas and selects a subset of them that are classified around the "user interaction area" focusing mainly on the development of new interaction mechanisms, new ways of presenting information and evaluation of devices. Most of these research papers in fact are technology-oriented and focus on how technology can be used in the AAL context instead of looking at the users' need and discuss their implications for practice.

Some results come from studies aimed at investigating seniors perception and monitoring needs with respect to the "smart home" technology devoted to improve QoL and/or monitor older users' health status $[15,43]$. Results of the first study showed a rather positive attitude toward devices able to enhance their lives. The main areas of intervention cited for these tools are emergency help, prevention and detection of falls, monitoring of physiological parameters. In the second study, both the elderly and their family were involved in order to identify monitoring needs and expectations. Themes like maintaining independence, detecting cognitive decline, sharing of information, and the trade off between privacy and usefulness of monitoring have been highlighted. Additionally, the acceptance by the elderly of unobtrusive in-home monitoring seemed to be closely tied to the perceived utility of data and services generated by such systems.

Both studies were mainly conducted using only qualitative research (focus groups) without involving a statistically significant numbers of participants well distributed among primary and secondary users. Additionally, the suggested domains of interventions for AAL systems were not deeply analyzed subdividing them into sub-items and no clear indications about a level of priority were provided to the systems developers.

One work that considers a large number of participants and combines qualitative and quantitative research has been carried out within the SAAPHO project [16]. In this work a statistically relevant numbers of older users from two different countries have been recruited for eliciting requirements of an intelligent system to support independent living. A number of monitoring needs (both related to the environmental safety and health status) emerged and the possibility to use the technology to facilitate contacts with relatives and friends was highlighted. This study provides qualitative indications from primary users on the sphere of intervention for AAL system not dwelling on the details of the difference in priorities of the specific services thus leaving space for improvement in the user needs elicitation and its implications for the system developers.

\subsection{Progress beyond state-of-the art}

The present study aims at assessing the users' needs for AAL services that perform older adults monitoring at home. The paper gathers users' feedback from a twofold perspective, namely the point of view of both older users at home directly exploiting the AAL technology and of their carers who also indirectly benefit from the AAL services while providing their care. The analysis is based on a methodology that combines qualitative and qualitative analysis [7]. The quantitative analysis enabled the production of a complete set of user requirements validated with a significant number of user representatives. A subsequent elaboration of the results synthesized a clear indication of the level of priority for the elicited services and a set of suggestions that can be useful for the practical development of innovative AAL services.

\section{New AAL services: The GIRAfFPlus concept}

The study described in this paper has been grounded on a project named GIRAFFPLUS ${ }^{1}$ [12], in which the authors have been involved. The aim of the project was to develop and thoroughly evaluate a complete system able to collect elderly people's daily behaviour and physiological measures from distributed sensors, perform context recognition and long-term trend analysis. The original idea was to provide a valuable system capable to support both the older user at home and their caregivers contributing to detect early signs of agerelated deterioration in the older adults. The main target of the conceived system are healthy older persons who are at risk of loosing their independence. Many projects focus on specific diseases dealing with services that can be useful for a specific class of users. For instance, the NOCTURNAL project [32] aimed to develop a tele-health system to support the nighttime needs of people with dementia. MATCH [1] is a research program that is supposed to support both users and companies in assessing the value of medical devices, focusing on a wide range of medical issues, and consequently on a different set of target patients. In contrast to these efforts, the GIRAFFPLUS's focus was to support prevention, thus contributing to

\footnotetext{
${ }^{1}$ http://www.giraffplus.eu
} 


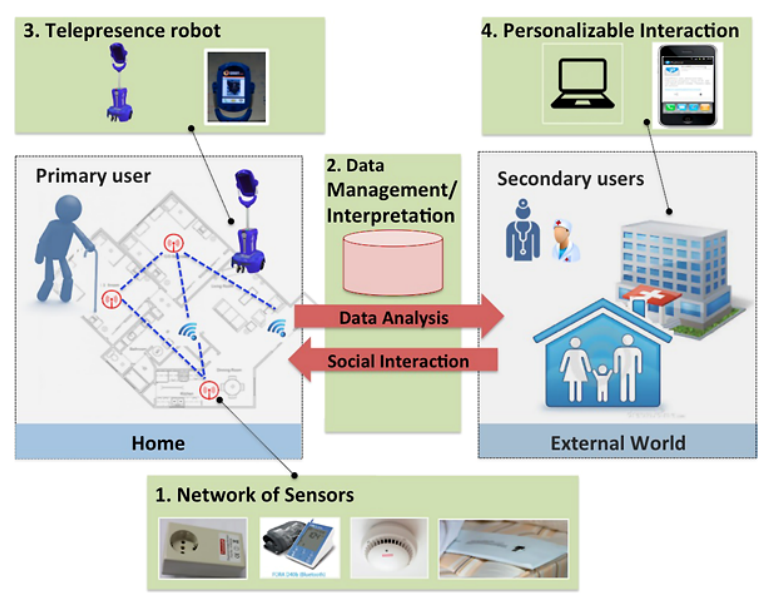

Fig. 1. The GIRAFFPLUS conceptual idea.

assess possible deterioration of health of people living alone. In this respect, the target users of the system are those people still independent, not suffering from severe medical conditions, but still at risk of becoming dependent.

The starting point of the GIRAFFPLUS project allowed an initial conceptualization of the system that is sketched in Fig. 1.

The picture introduces the key concepts relevant for the current paper that are: the typology of users, the technological components and examples of services we wanted to investigate.

\subsection{Typology of users}

First of all we can identify the human actors who are the target of the AAL services:

- The primary user who is the elder person (EP) living at home, mostly alone, that the system is supposed to actively support; In fact, the elderly play the main role of recipients of the services and monitoring from the intelligent environment.

- The secondary users, that is a network of people who participate in the support and monitor of the older adult from outside his/her home. This group benefits from the AAL system directly when using its services (at a primary end-user's home or remotely) and indirectly when the care needs of primary end-users are reduced. Usually, formal and informal secondary users may have different expectations from the system functionalities.

For this reason, in order to further investigate this aspect, we subdivide secondary end-users into two different sub-groups: (a) Healthcare Pro- fessionals, $H P$, an individual healthcare provider who may be a healthcare professional in medicine, nursing, or a field allied to health; (b) Caregivers. An informal caregiver, CG is a close relative or a friend who takes care of the primary end-user or in general has regular contact with him/her. A formal caregiver is a person trained to take care of the elderly especially with a social emphasis (rather than a medical one) on the type of support. Municipalities or social health cooperatives usually provide and train the formal caregivers.

\subsection{Key technological ingredients}

The key technological ingredients of the system (see Fig. 1) that constitutes the main building block of GIRAFFPLUS are: (1) a network of sensors deployed in the home that continuously gathers data both from the environment and related to the health of the person (e.g., blood pressure); (2) a data management and interpretation module that guarantees data storage and interprets data coming from both environmental and physiological sensors with the ultimate goal of synthesizing contextualized services; (3) a telepresence robot (the Giraff ${ }^{2}$ ) that guarantees communication between people outside the house and the primary user inside the house, enriching the dialogue with the possibility of moving in the home environment and performing visual monitoring through a camera connected to the robot [8]. The robot can also be seen as the focal point of attention for the user and a means through which the system can vehiculate the services at home to the older user; (4) a personalizable interaction front-end that allows to visualize data from the house to the secondary users, and also, to call the robot from outside the house.

It is worth highlighting the idea of a telepresence robot as a potential exploitable ingredient to allow secondary end-users to visit the assisted person in his/her home via an embodied robotic presence through which the visitor can communicate and at the same time move around in the environment. The robot is the technological component considered to be placed in the home of the elderly and used to connect to healthcare professionals as well as family members. This element of the system is mainly thought as a means to enable the delivery of services that are more "social oriented" and

\footnotetext{
${ }^{2}$ http://www.giraff.org.
} 
can also be potentially enriched with additionally services.

\subsection{Services}

Having described the key general elements of the GIRAFFPLUS we started conceiving some services for both the primary and secondary users, that are the main target of our investigation. An original list was synthesized, but the need emerged to validate and integrate them through a systematic study involving real users of both typologies. From the secondary user perspective example of services originally thought to be of value included:

- Long-term behavioural and physiological data analysis and monitoring. More specifically, a set of GIRAFFPLUS services is oriented towards the measurement of parameters of interest that may directly or indirectly indicate deterioration or decline of health i.e., early detection. The sensed physiological and environmental data, are monitored and intelligently interpreted in order to provide useful indications to caregivers.

- Producing reports that contains summarized data, and information about aspects of interest.

- Exporting data for a real time use (hence, for example, for issuing alarms). In fact, the system can rise alarms and/or send warnings, for instance, in case of falls or in case of abnormal physiological parameters.

Toward the primary users the project idea was to implement the following functionalities:

- The communication through the telepresence robot is the basic media for social communication from outside into the house through virtual visits.

- Having such a general set up, the system could offer a channel from the secondary to the primary for messages and reminders created through the visualisation front-end and delivered on the robot screen.

The messages and the information open up an interesting spectrum of possibilities that can be coordinated by personalization because different people may like different delivery of the information to each of the human actors connected to the dialogue with the system.

After this synthesis of initial services the effort was devoted to more critically and rigorously investigate the users' requirements for such kind of system by carrying out a rigorous investigation on users expectations.

\section{Elicitation of user needs}

A specific effort of the project has been then focussed on the user requirement analysis in order to both gather real users' needs and expectations with respect to the system and derive more general indications with respect to innovative AAL services. To this purpose 3-months of intense work have been dedicated to recruit representatives of both primary and secondary users and perform an intense investigation whose methods and results are described here.

\subsection{Objective and motivations}

The objective of our study was to gather users' needs and opinions with respect to the AAL services and to identify a set of services that the users (both primary and secondary) consider as most important to be realized. Specifically, the study aimed at:

- eliciting and validate what activities, events, physiological and psychological data or behaviours are important to observe;

- determining the level of usefulness and acceptance of this monitoring.

Overall we aimed at: identifying the set of factors that are particularly important to ensure early detection of possible deterioration of health; investigating what type of support could be useful to cope with agerelated impairments, and contributing to understand what is needed to support preventive medicine. These indications allow to combine the technological components previously presented and offer dedicated services that are grounded on real needs and expectations of users in terms of improving their independent living and/or to facilitate the roles of their carers.

\subsection{Method}

The general method of the study ${ }^{3}$ used to investigate the above-mentioned aspects is sketched in Fig. 2. It is based on a multi-dimensional approach that entailed three main directions of work:

1. Analysis of existing literature on relevant indicators for independent living.

\footnotetext{
${ }^{3}$ The general method has been conceived with the help of the School of Health and Medical Sciences of the Örebro University, the Lund University, the Andalusian Health Service and the Örebro County Council. In this paper we focus on the application of the methodology on a sample of Italian users.
} 


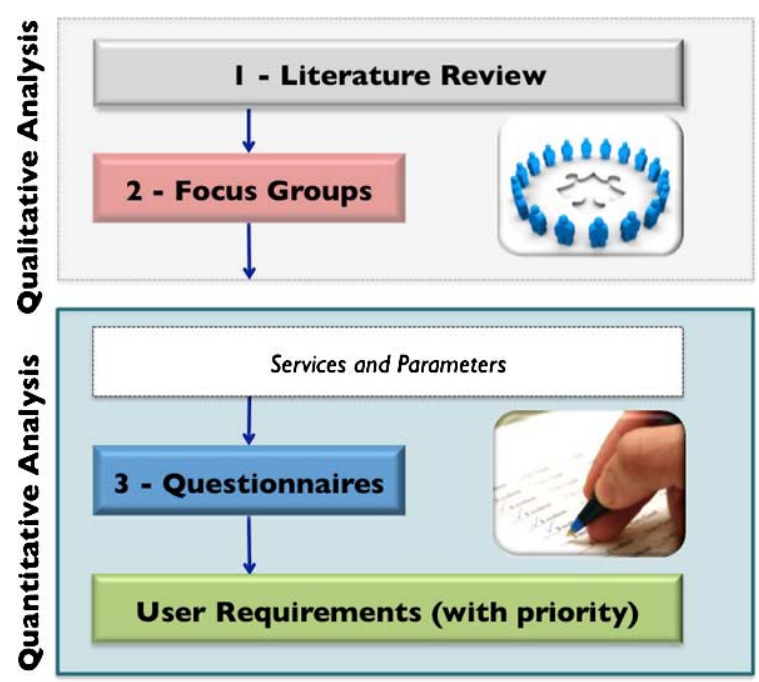

Fig. 2. General method for user requirement analysis: quantitative analysis is used to validate results and define priorities.

2. Qualitative study to identify user requirements for the GIRAFFPLUS system.

3. Quantitative study to validate and prioritize the user expectations.

The first step was devoted to study existing literature related to the main factors that contribute to endanger the level of independency of an elderly people at home. The idea was in fact to try to understand the relevant factors that represent a risk for the decline of elderly persons, and thus, should be monitored over time [9, pp. 13-17].

Starting from this, we then focused our effort on gathering feedback from real users (both primary and secondary), by involving them directly in giving their opinion on the services that an intelligent AAL system should offer.

To this purpose we combined qualitative and quantitative research as shown in Fig. 2, steps 2 and 3.

Specifically, a set of focus groups have been organized to define the services and parameters to monitor. A critical analysis of these requirements has then been made and used to create questionnaires to be administrated again to both primary and secondary users. The questionnaires directly reflected the list of services and parameters to be monitored that emerged from the focus groups work. The idea of these questionnaires was to have an indication on the level of perceived usefulness and acceptability of the services. These two measures were finally used to identify the different priorities for the user requirements (Step 3 of the study).
The rest of the paper reports the results of this analysis performed in Italy.

\subsection{Focus groups}

A focus group is a form of qualitative research in which a group of people are asked about their perceptions, opinions, beliefs and attitudes towards a product, service, concept or idea [41]. Two focus groups with primary and secondary users were conducted in Italy, in order to collect the perspective from users on their needs and what is important to observe to support independent living and assuring an acceptable QoL.

\subsubsection{Participants}

Table 1 shows the participants of the two focus groups performed in Italy.

Specifically a first focus group (FG1) involved representative of secondary users recruited among health care professionals (doctors, nurses, occupational therapists, physiotherapists, psychologist, home care professionals or other professionals) working in the field of care for elderly persons. The second focus group (FG2) involved elderly persons, as representative of the primary users of the GIRAFFPLUS system. Each group consisted of 10 persons. Health care professionals were recruited among medical doctors and paramedical staff working in the territory and involved in home care, or health professional working in cooperatives. Elderly persons were recruited among relatives of patients of Azienda Sanitaria Locale Roma A (ASL RMA).

As we mentioned earlier through this paper, the GIRAFFPLUS project is supposed to address both formal and informal caregivers needs. In order to better deal with this issue and to be better able to discriminate between the needs of these two typologies of caregivers, we involved a mixed group representing both formal and informal caregivers. Some of these participants

Table 1

Focus groups participants

\begin{tabular}{lcll}
\hline Focus group & Number & \multicolumn{1}{c}{ Users } & \multicolumn{1}{c}{ Observers } \\
\hline FG1 & 10 & $\begin{array}{l}\text { Medical doctors } \\
\text { physiotherapists } \\
\text { nurses } \\
\text { psychologists } \\
\text { health workers } \\
\text { sociologist }\end{array}$ & $\begin{array}{l}\text { 2 psychologists } \\
3 \text { engineers } \\
1 \text { psychiatrist }\end{array}$ \\
& & Elderly & 2 psychologists \\
& & 1 engineer \\
FG2 & & 1 psychiatrist \\
\hline
\end{tabular}


were both formal and informal caregivers (e.g., nurses taking care of their parents). In these last cases the underlying idea was to exploit both the professional expertise of these persons and their personal experience as caregiver of old parents or relatives they take care of. This approach allowed a focus on different needs due to different conditions.

Participants were recruited through ASL RMA and the focus groups meetings were held at the ASL premises under the supervision of the personnel working there thus adhering to the ethical code of that organization.

\subsubsection{Data collection}

A general description of the GIRAFFPLUS system was given to participants. After the presentation, a general schema ${ }^{4}$ to follow during the focus group has been adopted that was aimed at discussing which services, environmental events, information and behaviors of the person and physiological parameters are important to observe in order to ensure good quality of life and health for old people. Examples of questions for secondary users are: «... How do you think a smart home should be?»; «In order to detect early signs of psychophysical impairments, which kind of events are important to monitor in your opinion?»; «What kind of service you would like to receive from the system?»; «Which kind of person could benefit from living in a similar smart home?».

In contrast, quite different questions were asked to primary users such as: «If you had a smart home like the one just described, what kind of services should it provide?»; «What would you like to regularly monitor about your health status?»; «Which sensors are more useful for you to be used in a smart home?».

The moderators followed the discussion guide to generate interest in the topic, involve all the participants, and keep the discussion on track. One or more observers assisted the moderator. The role of the observers was to listen to the discussion, take notes and interact with the moderator when necessary. Notes from the observer could be used in directing the moderator in one way or another. Each focus group was a one and a half hour long discussion about health deterioration due to aging, elderly persons' expectations and need of support, how this support should be delivered, what should be monitored, and what consti-

\footnotetext{
${ }^{4}$ The general schema to follow during the focus group was conceived by the School of Health and Medical Sciences of the Örebro University, see also [9].
}

tutes an alarm. The focus group discussions were taperecorded and transcribed verbatim.

\subsubsection{Method of analysis}

The aim of the analysis was to identify examples and suggestions of activities, events, behaviours and physiological/psychological data to observe in order to detect deterioration of health and/or need for care or help. An additional aim was to identify the participants thoughts about the type of support services needed for the elderly persons.

The analyses were close to the direct wording in the text without deeper interpretation of latent meanings. The analysis was done by reading the text back and forth to get a picture of how the participants talked about different aspects of the identified factors. First of all, the text was read through to get a whole picture of the content. Secondly, suggested factors/activities/events/data that were mentioned by the participants was identified and marked in the text. The next step was to identify in the text if the focus group expressed that the marked data was an important factor to observe. For each factor the participants' motivation or arguments for this being an important factor were described. Furthermore, for each factor, discussions about needed support were identified and described.

\subsubsection{Focus group results}

The results of the focus groups have been organized according to macro-categories that seem to be relevant to consider for supporting both primary and secondary users. These categories are also in line with the International Classification of Functioning, Disability and Health (ICF) ${ }^{5}$ developed by the World Health Organization [44]. Specifically data were sorted according to the ICF chapters (first-level) and grouped as in the following sections.

Body functions and physiological parameters. Both primary and secondary users claimed that a decrease of body functions is something important and relevant to observe for early detection of health deterioration. Examples of things that could be important to monitor are: cardiovascular functions ([ “....For heart patients like me, heart monitoring is important”]), blood sugar, blood

\footnotetext{
${ }^{5}$ ICF is a global theoretical framework for classification of health and health related domains. It describes interactions between specific health conditions and activities, participation, body functions and contextual factors.
} 
pressure ([“...These sensors are all useful, but the medical ones are the most important, especially the ones that measures glucose and blood pressure"]), weight, body temperature, urinary incontinence/urinary infection and muscle weakness. These findings are also consistent with studies available in literature being the factors related to degree of independence and need for support [22].

Changes in daily activities and routines. Additional aspects that seem to be interesting to monitor are the possible changes in the "usual routines" of older persons at home. This was discussed mostly by the secondary users (caregivers). Detected changes in daily routines of the older person were described as possible signs of deterioration of health and increased need for support. Specifically the following aspects have been mentioned: loss of routines, inability to manage the daily activities, changed patterns of activity and mobility in the home, more time spent in bed than usual, leaving home at unusual times, mobility decline, number of visits to the toilet at night; eating and drinking deterioration; personal hygiene; ability to cope with diabetes, ability to and time spent on preparing food, forgetting to turn off the light or the stove. A specific attention has then been given also by elderly to the ability to handle medications. This result is supported by several references in the literature $[13,19,24,34]$ that describe how the ability to manage activities of daily living is important for independence and quality of life and also as an important detector of risks, for example risk of falls.

The one related to risk of fall is a recurrent and particularly relevant area identified in the focus groups especially with elderly users but also with caregivers. Both falls and fear of falling have been reported in both focus groups ([“...I would start first by monitoring falls in the house”]). In addition, people have expressed the concern that no one could find them when they have fallen. Also in the literature the risk of falls and the fear of falling are evident as a recurrent factor that is discussed in the relation to independence for elderly people [39].

Psychological and social factors. Both professionals and the older persons identified the fear of being alone and the need for contact with relatives and professionals ([ “... it is important to detect psychological status, to maintain contact between patient and health care staff or between patients after hospitalization”]). Additionally, regarding home care, ([ “... in addition to a phone call or a real visit this could be another way to be in contact"]). Expressions of isolation and anxiety were identified by professionals as early signs of decline.

Environmental factors. Both primary and secondary users mentioned the need to prevent potential risks related to environmental factors. Factors suggested to monitor were for instance: fire detection, taps opened, smoke detection, wet floor, temperature, and gas leaking. Another aspect that was mentioned by the elderly was the fear of intrusion in the home. This is also in line with our previous study [10] where the support for environmental safety have been suggested as one of the most important aspects in the home monitoring domain.

Overall primary users showed their interest in the potential of the system ([“... You are showing me some aids that seem necessary and useful to me"]), but they expressed also some concerns about privacy. In fact, people expressed also additional feelings about the system proposal. Older people recognized its potential usefulness and necessity in some cases, but they also pointed out concerns, in particular, with respect to the "monitoring issue". Indeed also the privacy issue has been raised. Concerns about continuous monitoring and access to the data were raised. More specifically the need to protect data and to also possibly tailor the data gathering to different needs was considered as an important aspect of the system.

Additional aspects also emerged from the analysis. The technical solutions should not replace human contact, rather they should be seen as a means to foster and promote human communication and support. Additionally it is important that the technical solutions support the individuals' autonomy and do not make them dependent on the technology itself.

Even though the questions asked during the focus group did not specifically contained questions related to the costs associated to the service as done in [40], participants raised concerns about possible additional costs they had to pay. In fact, keeping the costs of the service low is a key requirements for older adults. Additionally they also would expect the participation of the National Health Care service that might bear part of the costs. 
A final consideration, related mainly to the focus group with health professionals, was an expressed need to focus on a specific typology of assisted person in order to provide their feedback. This entails that different people have different needs and in turn suggests a user requirement for the solutions to be customizable to different cases.

\subsection{Quantitative analysis of user needs}

Starting from the focus group analysis we then reasoned on how this feedback could be translated into user requirements for the system that could be considered as representative of users' expectations of AAL solutions and how to assess a level of priority that could be useful for the system developers.

\subsubsection{Method}

The first step was to examine the main areas of interest for monitoring that emerged from the focus group results. These categories were then analyzed and reasoned upon. Figure 3 shows the results of this subsequent elaboration. Specifically we identified two macro-categories that contains the 4 sub-categories. The first one can be seen as mainly related to the Person monitoring while the second one is related to the monitoring of the Home environment. In the first case, the services are devoted to monitor parameters, activities or routines of the primary users, while the second case corresponds to services devoted to monitor environmental factors, that is the things happening at home.

The Person monitoring services contains these three main subareas:

- Physiology Monitoring: that is all the services devoted to assess the body function and physiological parameters and vital sign monitoring.

- Social Interaction Monitoring: that is all the services, mainly delivered through the robot, that can contribute to facilitate the social interaction of the older users or, more specifically the

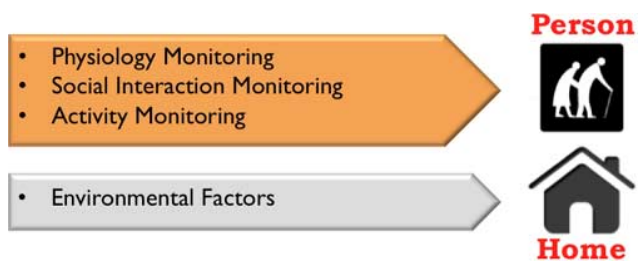

Fig. 3. Monitoring areas for the GIRAFFPLUS system. communication between primary and secondary users.

- ACTIVITY Monitoring: that is all the services that can be used to monitor changes in the daily routine of the person and in general facts or events that relate to the primary users.

The Home services are mainly related to the monitoring of ENVIRONMENTAL FACTORS.

Based on this classification we then translated the results of the focus groups into a list of services that the system could provide. More specifically the results have been organized into 4 different areas of interest (that consequently defined the different subscales of the questionnaires). Examples of statement describing the services and consequently the questions are: "Monitoring the movement of the person inside the house"; "Monitoring blood glucose levels in blood (glycemia)"; "Support the elderly person in remembering to take medicine or perform medication"; in the Figs 5, 6, 7 and 8 a synthetic version of the description of all investigated services (i.e., all items of the questionnaire) is provided that show the typology of questions that participants were asked to assess with respect to perceived usefulness and acceptance.

\subsubsection{Material}

The questionnaires to assess the perceived usefulness have been based on the following 5-point Likert scale:

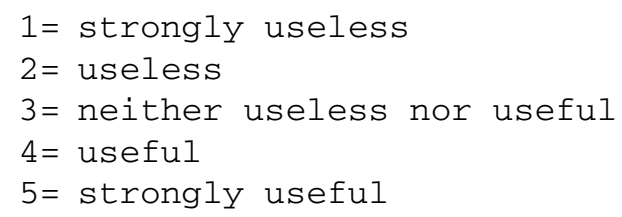

A similar scale was adopted to access the acceptability variable. In this light items were repeated in the questionnaires and participants were asked to rate also on acceptability according to the following scale:

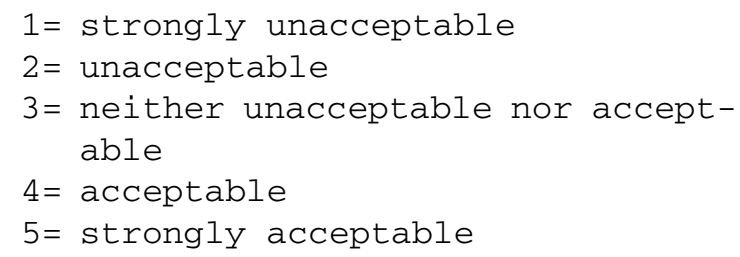

The overall questionnaire consisted then of 45 items for usefulness and 45 items for acceptability.

Internal consistency of the subscales has been assessed through the Cronbach's Alpha. The computed coefficients revealed good reliability for all the sub- 
Table 2

Crombach's Alpha coefficient for each subscales

\begin{tabular}{lcc}
\hline & Usefulness & Acceptability \\
\hline Physiology monitoring & 0.91 & 0.92 \\
Social interaction monitoring & 0.78 & 0.82 \\
Activity monitoring & 0.91 & 0.93 \\
Environmental factors & 0.82 & 0.78
\end{tabular}

scales both for usefulness and for acceptability (see Table 2).

Considering that questionnaires had to be administered to three different kinds of users (primary users and two different types of secondary users), different variants were developed in order to find the most suitable version according to the users' language and understanding. Specifically, the same questions were formulated in different ways depending on the user's typology. For primary users (older persons) the questions were developed avoiding technical terms. The application and response to the questionnaire was individual.

\subsubsection{Participants}

We recruited 105 persons for this evaluation phase. Specifically 44 primary users were the elderly people (EP), and a total of 61 secondary users participated in the study. More specifically, 33 informal caregivers (CG) were recruited among primary users' relatives or friends, and 28 Health Professional caregivers (HP) such as physicians, home care assistants, psychologists, nurses, and physical therapists. The elderly were for the most part women (74.4\%), and they were on average 79.5 years old $(\mathrm{SD}=7.32)$. The most prevalent educational level was quite low, in fact $51.3 \%$ of them attained just the primary school, and for the most part they were retired (92.3\%), and lived alone (48.7\%). When they were asked about their health status just few of them judged themselves as satisfied with their condition. In fact, only $18 \%$ rated their condition as completely satisfactory. Regarding the informal caregivers, 15 were females and 18 males. The mean age was 49.3 years old $(\mathrm{SD}=8.29)$. The most part of them attained the high school (54\%) and only $42 \%$ got a university degree. For the most part these persons were still working (96\%), thus having the need to arrange their job with their caring activities.

The subgroup Health Care Professional were composed by 16 females, and 12 males. They were on average 45.93 years old $(S D=10.71)$.

Elderly people were recruited mainly into day care centers (Associazione Arca di Noè and Virtus Italia). Care Givers were recruited with the help of the above
Table 3

Opinions of participants toward technology. Percentage scores

\begin{tabular}{lccc}
\hline & EP & CG & HP \\
\hline Completely negative & 0 & 0 & 3.6 \\
Quite negative & 0 & 0 & 3.6 \\
Neither negative nor positive & 12.8 & 3.1 & 0 \\
Quite positive & 69.2 & 43.8 & 50 \\
Completely positive & 17.9 & 53.1 & 42.9 \\
\hline
\end{tabular}

mentioned organizations and Health Professionals into ASL. All subjects were also asked about their attitude toward technology in general.

The whole sample expressed a positive attitude toward it, no one stated negative opinions, except for two health care professionals (see Table 3 for details).

\subsubsection{Statistical analyses}

Statistical analyses were conducted in order to assess different levels of perceived usefulness and acceptance for each parameter or event to monitor. More specifically, we relied on the analysis of the variance with one factor, three levels (Subjects - EP, CG, HP). First, the four sub-scales identified in Section 4.4.1 were considered, in order to investigate differences in general areas among subjects. Specifically, we wanted to investigate whether there are some monitoring areas considered as more important than others in order to support independent living. Subsequently, within each sub-scale, the single items were taken into account, in order to investigate different degrees of perceived usefulness of single parameters and events (items) to be monitored. Finally, in order to assess if usefulness is associated to acceptability, correlation analyses were performed. In particular, correlations among four usefulness sub-scales and acceptability sub-scales were calculated.

\subsubsection{Results}

The whole sample seems to perceive the overall system as a useful support to independent living, as the scores of the usefulness of the system were quite high (see Fig. 4). The categories Physiological Monitoring and Environmental Monitoring received the highest scores. Specifically, participants rated the usefulness of physiological monitoring as 4 mean score $(\mathrm{SD}=$ 0.65 ); the usefulness of home monitoring as 4.24 mean score ( $\mathrm{SD}=0.41$ ); the usefulness of person's activities monitoring as 3.80 mean score $(\mathrm{SD}=0.56)$ and the usefulness of facilitating social interaction as 3.73 mean score $(\mathrm{SD}=0.45)$.

When performing mean difference analyses among users, no significant differences emerged with respect 


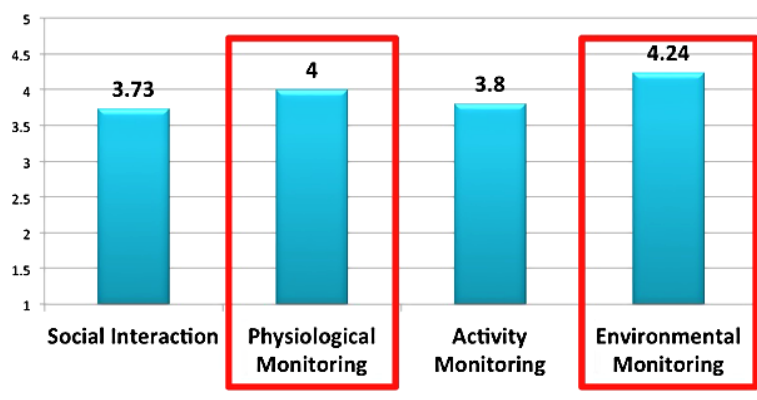

Fig. 4. Overall assessment of the AAL system per category: Means - scores 1-5.

to the perception of usefulness of the four sub-scales. This means that all monitoring areas are perceived by older people, caregivers and health professionals as equally useful. In order to test if gender could affect this perception, we also performed a mean comparison within each user group considering usefulness rating in the sub scales as independent variable. No significant differences emerged. This means that both males and females equally rated the usefulness on every monitoring areas.

After this analysis we also applied the Anova test on the data of each sub scale in order assess possible differences among the single individual items within each sub-scale.

Social interactions. Overall all items related to the ability of the system to support social interaction were rated as useful by the participants (see Fig. 5). The means values give a quantitative indication of the perceived usefulness for each specific monitoring service. Specifically, facilitating social interaction between the elderly and the physician (item 12), between the elderly people and their relative who takes care about him/her (item 24), and between the elderly person and the home care assistant (item 14) are considered all as useful. Also the possibility of issuing a report of the elderly person's day to a relative or to a trustworthy person (item 13), and even more the possibility of warning relatives in case of danger for the elderly (item 29) have been judged as important services. Finally, the reminding functions of the AAL system, such as reminders for medications or taking medicine (item 15), and the support for the contact between the doctors and the patients for monitoring physiological parameters after hospitalization or for any illness (item 11) seem to be important aspects to implement in AAL solutions. Nevertheless, giving

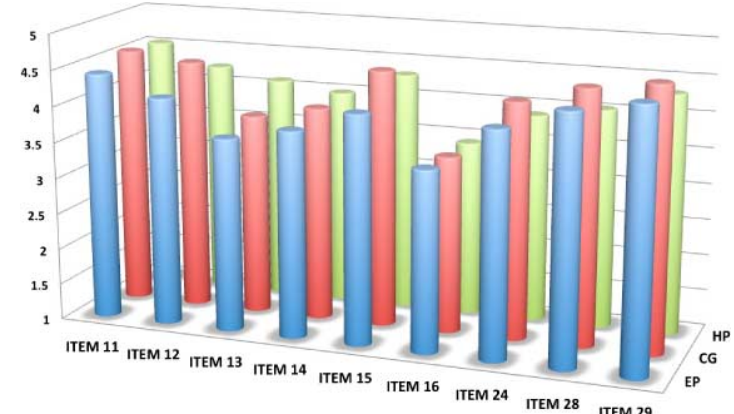

\begin{tabular}{l|lccc} 
Item & Description & EP & CG & HP \\
\hline ITEM 11 & Contact for monitoring physio parameters & 4.43 & 4.58 & 4.54 \\
\hline ITEM 12 & Facilitation of contact with the doctor & 4.16 & 4.48 & 4.25 \\
\hline ITEM 13 & Report of the day to a caregiver & 3.68 & 3.79 & 4.11 \\
\hline ITEM 14 & Contact with home care assistance & 3.86 & 3.97 & 4 \\
\hline ITEM 15 & Contact to remind or help in taking medication & 4.16 & 4.55 & 4.32 \\
\hline ITEM 16 & Contact a relative if lights are on during night & 3.5 & 3.45 & 3.43 \\
\hline ITEM 24 & Contact with family members & 4.11 & 4.27 & 3.89 \\
\hline ITEM 28 & Forced virtual visit in case of emergency & 4.41 & 4.52 & 4.04 \\
\hline ITEM 29 & Message to a secondary user in case of danger & 4.57 & 4.64 & 4.32 \\
\hline
\end{tabular}

Fig. 5. Social interaction area. Mean scores given by the different users (EP, CG and HP) to each item: Scale 1-5.

a direct notice to a relative if there are lights on during the night (item 16) seems not to interest the users, with no significant differences among users type. Only item 28 (forced entry by a close relative or a trustworthy person in case of emergency $\left.{ }^{6}\right)$ revealed significant differences among users $\left(F_{(2,104)}=3.75 ; p=0.027\right)$. More specifically caregivers $(M=4.52 ; \mathrm{SD}=0.57)$ rated this system service significantly more useful than health professionals $(M=4.04 ; \mathrm{SD}=$ $0.83)$.

The means values for each item and an explanation of the items are depicted in Fig. 5.

Person activities monitoring. The idea behind this set of services is to monitor specific activities of daily living that are indicative of well-being and autonomy of the elderly.

As shown in Fig. 6, primary users rated all services as less useful than secondary users on average.

Some of these differences resulted statistically significant. The significant results are related to

\footnotetext{
${ }^{6}$ This is a specific capability that allows one component of the GIRAFFPLUS system, i.e., the GIRAFF robot to force the usual operational functionalities. It enables an authorized client to bypass the old person authorization and connect with the home environment.
} 


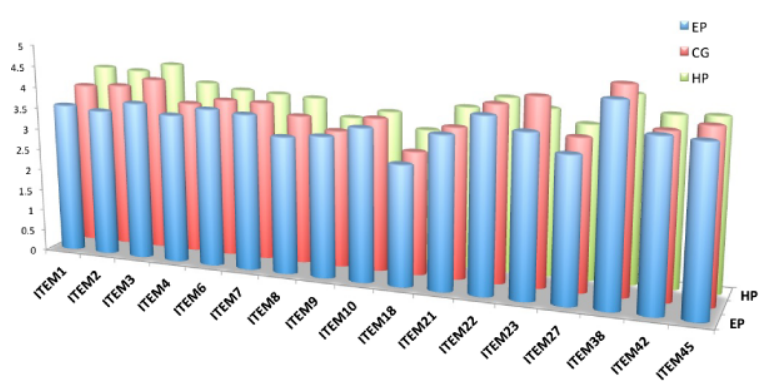

\begin{tabular}{l|lccc} 
Item & Description & EP & CG & HP \\
\hline ITEM 1 & Detection of the person's position at home & 3.55 & 3.85 & 4.14 \\
\hline ITEM 2 & Monitoring movements in the house & 3.48 & 3.91 & 4.11 \\
\hline ITEM 3 & Detection of absence of movements & 3.73 & 4.12 & 4.32 \\
\hline ITEM 4 & Temporal monitoring of persons' position & 3.52 & 3.61 & 3.93 \\
\hline ITEM 6 & Detections of changes in routines & 3.73 & 3.76 & 3.82 \\
\hline ITEM 7 & Monitoring person's activities during night & 3.68 & 3.76 & 3.79 \\
\hline ITEM 8 & Monitoring the ability to prepare meals & 3.23 & 3.52 & 3.76 \\
\hline ITEM 9 & Monitoring the time taken to prepare meals & 3.32 & 3.24 & 3.36 \\
\hline ITEM 10 & Monitoring time spent in the shower or bath & 3.59 & 3.61 & 3.57 \\
\hline ITEM 18 & Monitoring the frequency of use of the fridge & 2.84 & 2.91 & 3.21 \\
\hline ITEM 21 & Monitoring frequency of social interactions & 3.59 & 3.55 & 3.82 \\
\hline ITEM 22 & Detection of a decline in the mobility & 4.09 & 4.15 & 4.11 \\
\hline ITEM 23 & Detection of person's absence at unusual time & 3.8 & 4.39 & 3.93 \\
\hline ITEM 27 & Monitoring the use of home appliances & 3.39 & 3.55 & 3.64 \\
\hline ITEM 38 & Fall detection & 4.64 & 4.79 & 4.39 \\
\hline ITEM 42 & Detection of time spent in bed & 3.95 & 3.85 & 4 \\
\hline ITEM 45 & Monitoring the ability to maintain balance & 3.91 & 4.03 & 4.04 \\
\hline
\end{tabular}

Fig. 6. Person monitoring area. Mean scores given by the different users (EP, CG and HP) to each item: Scale 1-5.

the detection of the person's presence inside the home, i.e., detecting the position of the person inside the house (item $1 ; F_{(2,104)}=3.84$; $p=0.025)$, and monitoring both the movement (item $\left.2 ; F_{(2,104)}=5.19 ; p=0.007\right)$ and the absence of movement (item $3 ; F_{(2,104)}=3.70$; $p=0.028)$ of the person inside the house. Indeed, both these two items were rated more useful by health professionals than by elderly people who rated them significantly less important and useful on average. All the other items, which cover areas like persons' mobility and daily routine, were rated as useful by all respondents. The only exception is related to the nutrition capabilities, item 9 (monitoring the time taken to prepare for lunch) and item 18, monitoring the frequency with which the refrigerator is opened by the person. In fact respondents did not seem interested in these two types of services.

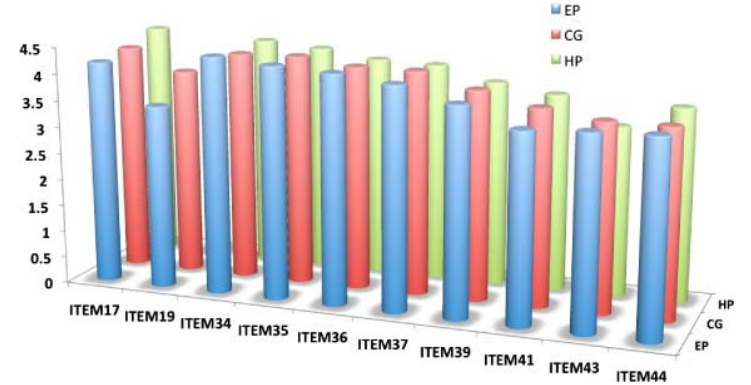

\begin{tabular}{l|lccc} 
Item & Description & EP & CG & HP \\
\hline ITEM 17 & Monitoring vital signs at night (breathing) & 4.2 & 4.27 & 4.46 \\
\hline ITEM 19 & Detection of body ?uids on sofas, beds, etc. & 3.45 & 3.89 & 3.82 \\
\hline ITEM 34 & Monitoring heart rate activity & 4.45 & 4.3 & 4.36 \\
\hline ITEM 35 & Monitoring body temperature & 4.36 & 4.33 & 4.28 \\
\hline ITEM 36 & Monitoring blood glucose levels (glycemia) & 4.3 & 4.21 & 4.14 \\
\hline ITEM 37 & Monitoring blood oxygen levels (oximetry) & 4.18 & 4.21 & 4.11 \\
\hline ITEM 39 & Monitoring body temperature & 3.91 & 3.94 & 3.86 \\
\hline ITEM 41 & Determining episodes of incontinence; & 3.55 & 3.7 & 3.71 \\
\hline ITEM 43 & Monitoring the person body weight & 3.61 & 3.55 & 3.21 \\
\hline ITEM 44 & Monitoring the person's sleep & 3.64 & 3.55 & 3.64 \\
\hline
\end{tabular}

Fig. 7. Physiological monitoring area. Mean scores given by the different users (EP, CG and HP) to each item: Scale 1-5.

Finally, the main concern seems to be connected with the possibility of the system to be able to determine if the person falls to the ground (item 38). In fact, all the respondents rated this feature as extremely useful with the higher score given by caregivers.

Physiological monitoring. Also for the physiological monitoring usefulness, an analysis on each single service was performed. No significant differences among subjects were found. In fact, elderly people, caregivers, and health professional gave a similar average score on the usefulness of this type of services.

Specifically, Fig. 7 illustrates how they all considered each service or parameter monitoring as useful. Nonetheless, monitoring parameters that represent vital signs or age-related diseases, such as hearth functions (item 34), blood pressure (item 35), glycaemia (item 36), and oximetry (item 37) obtained higher score. This is also true for monitoring such parameters during the night (item 17).

Home monitoring. The environment monitoring is considered by every respondent as an important service that an AAL system could offer.

Figure 8 shows that services referring to the $d e$ tection of potentially dangerous environmental 
situations (item 5) were rated as more strongly useful to monitor. Specifically, gas leaks detection (item 30), detection for presence of risky situations for the development of fire (item 32), and detection of water leaks (item 33) were considered strongly useful to be integrated within the system.

Usefulness and acceptability. As depicted in Table 4, results show significant positive correlations between usefulness (U) and acceptability (A) in every sub-scale.

We may infer that participants perceived as acceptable what they consider useful with respect to

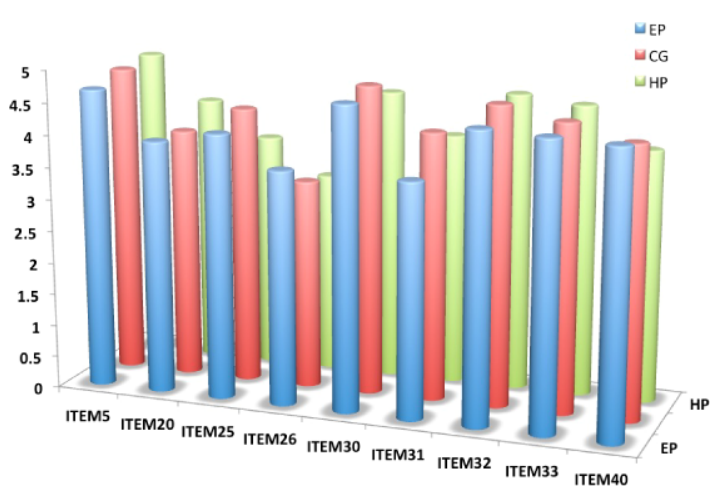

\begin{tabular}{l|lccc} 
Item & Description & EP & CG & HP \\
\hline ITEM 5* & Detection of danger (i.e. gas leaks, risk of ?re) & 4.68 & 4.82 & 4.89 \\
\hline ITEM 20 & Detection of objects that could cause falls & 3.95 & 3.91 & 4.21 \\
\hline ITEM 25 & Detection of open doors in the house & 4.14 & 4.33 & 3.68 \\
\hline ITEM 26 & Detection of lights on in the house & 3.66 & 3.27 & 3.14 \\
\hline ITEM 30 & Detection of gas leaks & 4.73 & 4.82 & 4.54 \\
\hline ITEM 31 & Monitoring extreme temperatures & 3.66 & 4.18 & 3.93 \\
\hline ITEM 32 & Detection of risk of ?re & 4.5 & 4.67 & 4.64 \\
\hline ITEM 33 & Detection of water leaks that may cause ?oods & 4.45 & 4.48 & 4.54 \\
\hline ITEM 40 & Detection of taps left open (water in the ?oor) & 4.41 & 4.24 & 3.93 \\
\hline
\end{tabular}

Fig. 8. Home monitoring area. Mean scores given by the different users (EP, CG and HP) to each item: Scale 1-5. every area supported by the system. This means that the more a service is considered useful, the more it is accepted by users.

Priority of functionalities. Considering the feedback gathered from users and presented above, a further refinement has been performed in order to assign a specific priority to each functionality and, then, elicit an additional valuable indication for system design phases. Specifically, three different priority levels have been considered: KEY, DESIRABLE and OPTIONAL.

A requirement is assigned a KEY level of priority when at least two end user types (i.e., EP and either CG or HP) estimate the associated item as useful (point 4 in the scale). For instance, within Person activity monitoring, detecting a decline in the mobility of the older person (item 22) is a key requirement since all end user categories assessed such functionality as useful, i.e., MEP $=4.09$, $\mathrm{MCG}=4.15$ and $\mathrm{MHP}=4.11$. This means that a KEY priority is assigned to user requirements whose utility is perceived as important by both the older person and a caregiver (being a professional caregiver or not), i.e., a potential positive effect is for both kind of users. A requirement is DESIRABLE when at least one end user type evaluates the functionality as useful. An example is Monitoring extreme temperatures (item 31) in Home monitoring category considered useful by caregivers $\mathrm{MCG}=4.18$. This means that a DESIRABLE priority is assigned to user requirements whose utility is perceived as important by either the older person or a caregiver with a potential positive effect for only one kind of users. Finally, a requirement is OPTIONAL when none of the user category assessed the functionality as useful. A clear optional requirement is monitoring body weight (item 43) in Physiological monitoring, since it is not considered useful by any

Table 4

Correlation between usefulness and acceptability in each sub-scale

\begin{tabular}{|c|c|c|c|c|c|c|c|c|}
\hline & 1 & 2 & 3 & 4 & 5 & 6 & 7 & 8 \\
\hline (1) U_COMMUNIC & 1 & & & & & & & \\
\hline (2) U_PHYSIOL & 0.606 & 1 & & & & & & \\
\hline (3) U_PERSON & 0.657 & 0.734 & 1 & & & & & \\
\hline (4) U_ENVIRONM & 0.640 & 0.558 & 0.597 & 1 & & & & \\
\hline (5) A_COMMUNIC & 0.752 & 0.445 & 0.559 & 0.564 & 1 & & & \\
\hline (6) A_PHYSIOL & 0.524 & 0.743 & 0.703 & 0.465 & 0.614 & 1 & & \\
\hline (7) A_PERSON & 0.584 & 0.646 & 0.845 & 0.523 & 0.653 & 0.796 & 1 & \\
\hline (8) A_ENVIRONM & 0.550 & 0.438 & 0.458 & 0.710 & 0.706 & 0.499 & 0.563 & 1 \\
\hline
\end{tabular}


Table 5

Functionalities priority distribution

\begin{tabular}{lccc}
\hline Category & KEY & DES & OPT \\
\hline Social interactions & 5 & 3 & 1 \\
Person activities monitoring & 2 & 6 & 9 \\
Physiological monitoring & 5 & 0 & 5 \\
Home monitoring & 4 & 4 & 1 \\
Total & 16 & 13 & 16 \\
\hline
\end{tabular}

of the end users. The OPTIONAL priority means that the service is not perceived as really useful.

This kind of analysis generated a mapping from functionalities into classes of priorities. A synthetic description of the distribution of such classification is depicted in Table 5 .

A detailed description of priority assignment is depicted in Table 6. It is worth noting that the high number of optional priorities assigned in both person activities and physiological monitoring is mainly related to privacy issues, i.e., users do not feel as useful/acceptable to have some vital signs (e.g., weight) or activities (e.g., time spent in bathroom) monitored.

\section{Discussion and implications for system design}

The investigation presented above was aimed to identify and validate the type of parameters, events, or situations particularly important to be detected and/or monitored in order to support elderly people at home through intelligent technology and AAL solutions. The goal was also to elicit a set of crucial features for AAL systems addressing specific issues within each ICF area in order to support the prevention of early deterioration of healthy conditions, and to promote independent living for the elderly. The assignment of priorities to each of these features has been set to influence the design, development and deployment of the GiraffPlus solution and, thus, enhancing its potential efficacy. The rest of this section presents a discussion addressing different macro categories and relating results (and assigned priorities) to the analysis of practical implications for the system. A set of significant scenarios has been defined to present concrete situations (i.e., relevant use cases) in which the users of the system can leverage its features.
Table 6

Functionalities priority assignment

\begin{tabular}{lc}
\hline Functionality & Priority \\
\hline & \\
Contact for monitoring physiological parameters & KEY \\
Facilitation of contact with the doctor & KEY \\
Report of the day to a care giver & DES \\
Facilitating contact with home care assistance & DES \\
Support the person in remembering to take medicine & KEY \\
Notice if there are still lights on during nights & OPT \\
Facilitating contact with family member & DES \\
Forced entry in case of emergency & KEY \\
Warning of danger to a caregiver & KEY
\end{tabular}

Person activities monitoring

Detecting the position

DES

Monitoring the movements

DES

Detection of the absence of movement

DES

Temporal monitoring of position

Detection of changes of habits

Monitoring of a person during night

Monitoring a ability to prepare lunch alone

Monitoring the time taken to prepare for lunch

Monitoring of time spent in the shower/bath

Monitoring the frequency the refrigerator is opened

Monitoring the frequency of social interactions

Detecting a decline in the mobility of the person

Detection of person's absence at unusual hours

Monitoring of the use of the stove in time

Determining if the person fall to the ground

Monitoring the person keeps staying in bed

Monitoring the person's ability to maintain balance

Physiological monitoring

Monitoring of vital signs during the night

OPT

Detecting the presence of body fluids

Monitoring heart rate activity

Monitoring body temperature

Monitoring blood glucose levels in blood

Monitoring blood oxygen levels

Monitoring body temperature

Determining episodes of incontinence

Monitoring the person body weight

Monitoring the person's sleep

Home monitoring

Detection of dangerous situations

OPT

OPT

OPT

OPT

OPT

OPT

OPT

OPT

KEY

DES

OPT

KEY

DES

DES

KEY

KEY

KEY

KEY

KEY

OPT

OPT

OPT

OPT

Detection of misplaced objects

KEY

DES

Detection of open doors

DES

Detection of lights on

OPT

Detecting gas leaks

KEY

Monitoring extreme temperatures

DES

Detecting development of fire KEY

Detecting water leaks $\quad$ KEY

Detecting taps left open
DES 


\subsection{Practical implications for a real AAL system}

An important aspect that emerged from the study is a general good attitude towards the use of AAL technology. Both primary and secondary users expressed positive feelings toward the technology. Specifically, both during the focus groups, and through the questionnaires administration, positive impressions emerged with respect to the proposed intelligent system. No negative opinions on the system and services emerged from the entire sample. At the same time, investigating the relation between the system's usefulness and acceptability, interesting results emerged. People seem to be confident in what they consider useful. A positive correlation between these two dimensions was found which might mean that people are not so distrustful of this kind of support. In other words, the perceived benefits that intelligent AAL solutions could bring to the elderly, seems to mitigate the negative effect of other concerns like privacy or mistrust. Nevertheless, a subtle issue about privacy still emerged. Especially in those cases where elderly people (primary users) had to deal with aspects related to their own activities monitoring, the expressed judgments were slightly less positive than the ones of the other participants (secondary users).

This consideration could be interpreted as a comprehensible concern about privacy and fear of being controlled by other persons. It is worth underscoring how this aspect should not be underestimated when building any kind of assistance and support technology. The emerging discussion about privacy has implications also for the system development. A twofold perspective could be adopted: (a) service perspective and (b) data policy perspective.

Implications for practice (IP) 1a: For the first aspect, this study suggests that an ideal system should be customisable so as to respect the user's preferences in terms of "level of invasiveness" (i.e., the user should be able to decide the level of monitoring invasiveness). Consequently, also the offered services may be tailored according to the availability of data. This entails to ensure service personalization.

Implications for practice (IP) $\mathbf{1 b}$ : From the second point of view, the relevance of implementing suitable data policies within the technical requirements for the design of an AAL system should be taken into account. In particular: the system shall protect stored data related to the old people activ- ities against accidental and/or unlawful destruction/loss/alteration as well as unauthorized or unlawful storage, processing, access or disclosure; it shall allow access to personal data only by authorized personnel and only for legally authorized purposes. Reliable security mechanisms for both storage and communication processes should be implemented and a clear and effective data access policy should be defined to avoid not proper access to data.

The IP is consistent with the data protection directive (Directive 95/46/EC), EU regulation that applied during the project and that it is still consistent with the new General Data Protection Regulation (GDPR) (Regulation (EU) 2016/679). The EU GDPR has been adopted on 27 April 2016 and will apply from 25 May 2018.

In addition to the general aspect, different action areas have been considered within the study, which includes social interaction, physiological data detection, environmental and person activities monitoring. All these areas of support have been considered as relevant by all participants. This result suggests the importance to develop a comprehensive solution to support elderly, trying to cope with their frailty in a comprehensive and pervasive way. Safety (both environmental and physical) represents a crucial issue to address as also already found in [10]. Indeed, monitoring dangerous situations at home, and monitoring vital sign was found to be very important. Results suggest how the ability of the system to handle critical events both real (e.g., risk of fire) and perceived (i.e., fear of falling) is particularly important and appreciated.

\subsubsection{Social interactions monitoring}

Results from the literature review show that social interactions is perceived as a means to contribute to a good QoL and better health for the elderly. Maintaining relationships, especially with family and friends is considered very important. Loss of relations might on the contrary lead to poorer health [4]. In line with this consideration, results of our study show that facilitating communication between the elderly and relatives is a useful service both for primary and secondary users thus suggesting that this could be a relevant service that an AAL system should provide. At the same time also communication with doctors, or home care assistants emerged to be a relevant issue to improve through the system. In fact, another way to exploit the "social/communication ability" of the system, seems 
to be its use to facilitate the doctors' work after hospitalization.

Overall, it clearly emerged that after an hospitalization period, patients usually feel abandoned and manifest the need to maintain a contact with their care givers. The AAL system could then be seen as a means to continue and prolong the monitoring and interaction period started at the hospital and then activated from home thus allowing a continuous monitoring of both physiological parameters and psychological conditions. Indeed, all users participating at the survey considered it as a strongly useful service.

IP 2: The presence of a technological component for enabling remote social interactions, such as the telepresence robot or an alternative solution constitutes a crucial element in an AAL system. In fact, the presence of such a technology can facilitate social interactions bridging the gap between primary and secondary users who live far from each other or, also, when physical visits are not possible. Additionally, when the old person is not able to go outside the house (e.g., due to a physical condition) a technology enabling interactions with the external world can play the role of a social hub potentially fostering interactions with relatives, friends or healthcare professionals.

More in details, informal caregivers found also particularly interesting and useful the possibility to have access to the living environment through the system and "virtually enter" into a house from remote locations (by means of the telepresence robot). This confirms the importance for relatives (or even friends) to provide assistance as promptly as possible. Indeed, in case of emergency, e.g., when a old person falls in the house, the robot would allow to remotely visit the house, to check the actual situation as well as to provide some initial comfort to the person and, then, to be able to inform in advance paramedics about the situation in case their intervention is required.

\subsubsection{Warnings and periodic reports}

Other specific functions connected to the one just mentioned are considered as useful. For example, all users recognized the usefulness of the possibility to get a warning or alarm from the system in case of dangerous situations detected by the sensors.

IP 3: A set of warning/alarm services should be implemented in an AAL system considering also redundant channels (e.g., pre-registered phone calls, SMS, emails, etc.). This is crucial to inform secondary users about potentially risky situations that may occur in the house and thus enhancing the safety condition for the old person. Additionally, the system could also guarantee the possibility to receive every day a sort of report on the elderly activities/status in order to reassure caregivers and relatives on the fact that everything went right during the day. The AAL system is then supposed to process and analyze the data gathered by sensors in the houses in order to produce concise information and generate daily reports containing aggregated and synthetic view of information for secondary users. Such reports should be generated taking into account also the relevant perspectives related to the different kind of users. In general, reports should provide different information to healthcare professionals or relatives and friends. In this sense, personalized services are crucial in order to adhere to users expectations.

\subsubsection{Activity monitoring}

A pervasive positive opinion about the monitoring mobility and balance decline has been found. The whole sample rated these services as particularly useful. A further confirmation of this result is also that participants unanimously rated the "detection of the person position", "person motion", and "motion absence" within the house, as a useful service. In this light detecting changes of body position in order to predict disability could be particularly useful. Different categories of users evaluated these functions slightly differently. Specifically elderly people found them less useful than secondary users. This finding can be interpreted as a fear perceived by the elderly to be "invasively monitored". The idea to be monitored in terms of movement inside their house does not seem to be particularly appreciated, even if they rated it as a useful issue. Some household tasks that contribute to maintain independence were also investigated. Specifically, the ability to prepare food has been taken into account since it can be considered as predictor of physical decline, survival, and quality of life. Specifically, cognitive impairment is an important factor related to poorer nutritional status and increased need for assistance and care [34], and good nutritional status is important to preserve independent living [35]. In this respect, three kinds of detections were considered: the ability to prepare food, the time taken to do it, and the frequency with which the person opens the refrigerator. Respon- 
dents seem not to consider these aspects as important for the system implementation. Maintaining a routine in daily activities seems on the contrary to be important. In fact, the detection of changes of person's habit in the daily activities inside the house (e.g., the person wakes up too late with respect to usual standards; the absence from the house is detected during unusual hours, e.g., overnight), can be seen as early sign of potential decline. In line with these findings the whole sample rated this type of service as useful recognizing its value in supporting prompt interventions in case of anomalies. Moreover, it is important to highlight that the main concern observed among elderly regards falls and fear of falling. In fact, monitoring possible person's falls is the item that received the highest score from the whole sample.

IP 4: A suitable set of environmental sensors should be constituted by typical telecare sensors, i.e., a set of binary sensors capable of sending signals to alert that a threshold has been crossed. Considering users preferences related to monitoring motion and position at home, a suitable set of sensors can be the following: (a) Fast Passive InfraRead (PIR) motion detectors for monitoring of movement, (b) bed/chair occupancy sensors for monitoring location in the house, (c) doors usage sensors for their opening and closing, and (d) electrical usage sensors for monitoring appliances utilization. Moreover fall detection sensors are also to be considered. The AAL system should also deploy an intelligent monitoring process service capable of analyzing and infer the actions performed by the user in the home processing the amount of data generated by such sensors. Then, the system should be also able to generate more meaningful information about activities and performing high-level reasoning over them. Then, inferred data can be exploited also to monitor activity patterns and generate alerts in case deviations from usual behaviors are detected (e.g., the system should detect cases in which an electrical device, e.g., a toaster, is forgotten switched on).

\subsubsection{Vital parameters monitoring}

Concerning body functions, literature evidences showed that several different functions might be risk factors of increased dependency. This means that detecting specifics changes in body functions could help both in preventive actions and in risk situations. Also disturbed sleep is associated with decreased ADL ca- pacity, risk of falls and poor quality of life $[31,45]$. In this line, monitoring the person's sleep, both in terms of motion (e.g., if he/she moves a lot during the night this could be a sign of a disturbed sleep), and in terms of vital signs (e.g., heart rate, breathing etc.), was considered by the whole sample as a useful function for AAL systems. As regard physiological monitoring, people were asked to answer about usefulness in monitoring specific parameters like blood pressure, glycaemia, oximetry, and body temperature. All participants rated all parameters as particularly useful. These results are also in line with evidences in literature. For example, research suggests that decreased functions of the cardiovascular systems are risk factors of decline in ADL and recurrent falls [21], and these kinds of detections may help in evaluating early signs of dysfunction. Overall the findings about useful in detection of incontinence situations are also in line with evidences that problems of urinary function are associated with increased dependency in ADL and risk of falling [45]. A similar trend is the result related to the monitoring of the presence of body fluids such as urine, blood, or sweat on sofas or beds, which have been considered as a useful function in the system by all participants.

IP 5: AAL systems should be endowed with telemonitoring medical devices enabling the possibility of storing assessments of vital parameters. An AAL system should be then capable of detecting and storing biomedical parameters (e.g., blood pressure, blood glucose, weight, oxygen saturation, temperature, among others) and providing that information both for reporting and high-level reasoning.

\subsubsection{Home environment monitoring}

Environmental factors resulted to be predictors of independent living functioning among elderly people. Such type of monitoring functionality might improve both social functioning and a feeling of safety. This means that people valued the home highly and that living in their own place could contribute to good quality of life $[4,28]$. In this direction, when asking to people about the usefulness of the detection of dangerous environmental situation, all participants recognize it as strongly useful. Similar results have been obtained about detection of gas leaks, and risky situations for the development of fire detection.

IP 6: In addition to telecare sensors discussed above, an AAL system should be equipped with a further set of environmental sensors to specifically 
address dangerous situations such as the ones indicated above. Among the sensors in the ADLife product line, some support a wide range of risky situations such as fire detection, flood detection and gas leak detection sensors. These sensors can generate suitable information to raise alarms and/or warnings about risky conditions and unsafe situations that may occur in the living environment and they can fully support home monitoring enabling an AAL system to ensure safety conditions.

\subsection{Relevant $A A L$ scenarios}

The analysis of results also allowed envisaging a set of possible scenarios as particularly relevant for the considered AAL domain. This section describes a sketch of three possible scenarios that were recurrent in the focus group analysis and, thus, judged as particularly appropriate to inspire the use case definition. Such scenarios show concrete situations in which users (both primary and secondary users) may leverage system features taking advantage of their capability. Such scenarios constitutes a set of significant use cases to show how the system effectively address actual needs requested by users and, then, to provide technology providers and services developers with concrete reference to design and develop the actual AAL system. For each of the three scenarios, a set of information is provided: a brief description, the type of users involved, the possible role of the AAL system, the sensors and the main parameters to be monitored and relevant to the case study and, finally, the more relevant user requirements and implications for practice (IP) discussed above.

\subsubsection{Monitoring a physiotherapy protocol}

A first scenario is constituted by the case of an elderly user following a protocol for physiotherapy rehabilitation. In Fig. 9, a schematic idea of the scenario is depicted. The envisaged primary user is an old person at home following a physiotherapy treatment protocol while the secondary user is a physiotherapist in charge of monitoring the elderly during the rehabilitation period. In a rehabilitation protocol, the physiotherapist aims to fully restore the ability to move in a patient. In this perspective, the physical therapist is supposed to assign to the elderly a rehabilitation protocol strictly related to his/her physical condition. The system can register relevant information such as for example: how much time during the day the patient spends in bed,

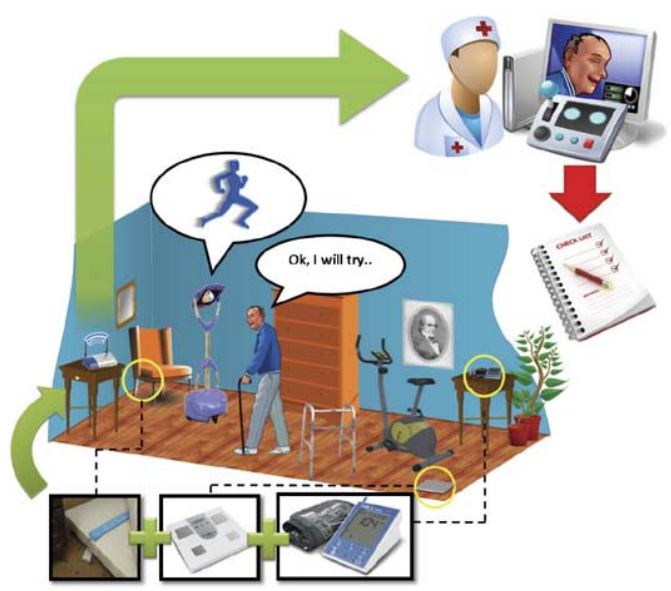

Fig. 9. Scenario 1: monitoring a physiotherapy protocol.

sitting, standing and moving positions. Such information can be provided periodically (e.g., on a daily basis) to the physiotherapist at the level of detail or as a cumulative data. In this way the therapist is able to gather an objective measure of the physical activity of the patient and possibly correct the protocol itself. Indeed, the time spent in certain activities can be considered as "normal" only within certain limits. Thus, collecting data over a certain period would also enable the therapist to perform an assessment of the user reactiveness to the therapy. Another important service is to enable the possibility of performing "Rehabilitation Session" exploiting the "telepresence" robot, i.e., having the physiotherapist connected through the robot to assist the primary user while performing rehabilitation exercises, assessing them and providing suggestions/comments remotely.

The relevant sensors needed in this specific case are mainly related to environmental parameters dedicated to localize the position and the status of the user in the house, while a telepresence robot could be exploited to implement the rehabilitation sessions. In general, the principal requirements involved in the scenario are related to the need to facilitate contacts between the person and healthcare professionals, to monitor the primary user position in the house, to provide periodic reporting to a secondary user and to support the detection of changes in primary user habits.

This scenario requires to provide a proper data policy to implement suitable privacy issues management for sensible data about the health condition of the primary user (IP 1) as well as increase the frequency of interactions between the physiotherapist and the older person through the robot (IP 2) and hopefully increas- 
ing the effectiveness in therapy monitoring. Then, the generation of periodic reports can help the physiotherapist in being aware about the actual conditions of the older person (IP 3). The deployment of intelligent monitoring techniques can actually support the detection of specific issues such as, for instance, incorrect adherence to therapy prescription would support early detection by the system of specific issues (IP 4).

\subsubsection{Monitoring after de-hospitalization}

One of the recurring situations among the elderly is related to de-hospitalization periods, who are discharged from hospital to return in their home and do not receive regular and continuous support by medical staff. In such cases, the problem is usually constituted by the inability/difficulty to maintain a constant and frequent contact between formal caregivers and the elderly person, sometime also worsened by the fact that many elderly people also have few relatives that can take care of them at home. Figure 10 provides a schematic idea of such scenario: an AAL system could act as a facilitator for such monitoring.

The primary user is an elderly person at home after a period spent in hospital while the secondary users are the medical staff monitoring physiological parameters as well as psychologists and/or social operators monitoring the psychological condition. The assisted living system can support the users in two different directions. As a support for psychological monitoring: the telepresence robot can contribute to maintain a continuum with the care and support received in the hospital, also ensuring that the monitoring service is more frequent. Obviously the monitoring service results as a

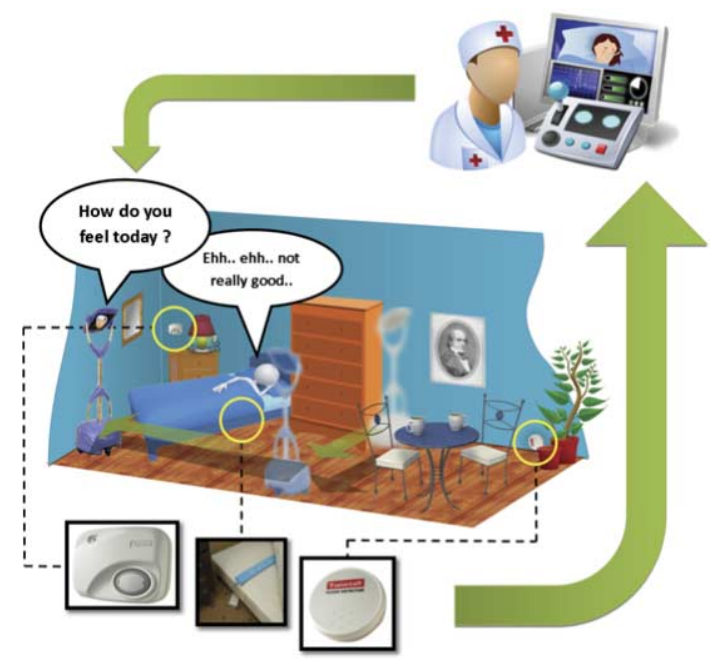

Fig. 10. Scenario 2: monitoring after de-hospitalization. combination of remote (through the robot) and physical assistance (through real visits). Health monitoring: the system allows monitoring of vital parameters after de-hospitalization, in particular those specifically connected to the given de-hospitalization condition (e.g., related to a surgery or a specific pathology). Moreover, allowing continuous and frequent contact, a doctor can also better assess a therapy protocol and, in case of need, plan for changes or ways to better customize it. Thus, the aim is to check whether the post-hospital period proceeds properly and, if necessary, to support a doctor with evidences that may lead to changes in the treatment.

The relevant sensors and parameters are mainly related to physiological monitoring, and again, the robot for remote communications. The principal requirements involved in this scenario are to facilitate contact between the person and healthcare professionals, to provide medical support and to monitor the vital signs of the user.

Proper data policy and privacy issues management is a crucial aspect also here (IP 1). Remote assistance through the telepresence robot is an essential aspect (IP 2) to implement a continuous care and close monitoring of older person conditions. Period of dehospitalization for elderly may constitute a really delicate situation and thus the possibility for secondary users to receive warning and/or periodic reports about his/her status can help in tightly monitor the actual conditions of the older person (IP 3 and IP 5).

\subsubsection{Daily activity monitoring by an informal caregiver}

The third scenario envisages a (general) daily monitoring of the elderly person by an informal caregiver (e.g., a son or a close relative). In Fig. 11, a schematic idea of the scenario is provided in which the technology can constitute a means to detect risky situations as well as provide secondary users with warning messages.

The involved primary user is an old person living alone at home and assisted only by a secondary user, i.e., an informal caregiver, e.g., a son or a close relative of the elderly people who takes care of him/her.

In this scenario, the role of the AAL system is to detect risky situations such as falls and, according to pre-defined emergency protocols, the system is to react providing the related secondary user with a warning/alarm message. Additional relevant services can be, for instance, to perform monitoring of some environmental events and, more in general, to infer the ac- 


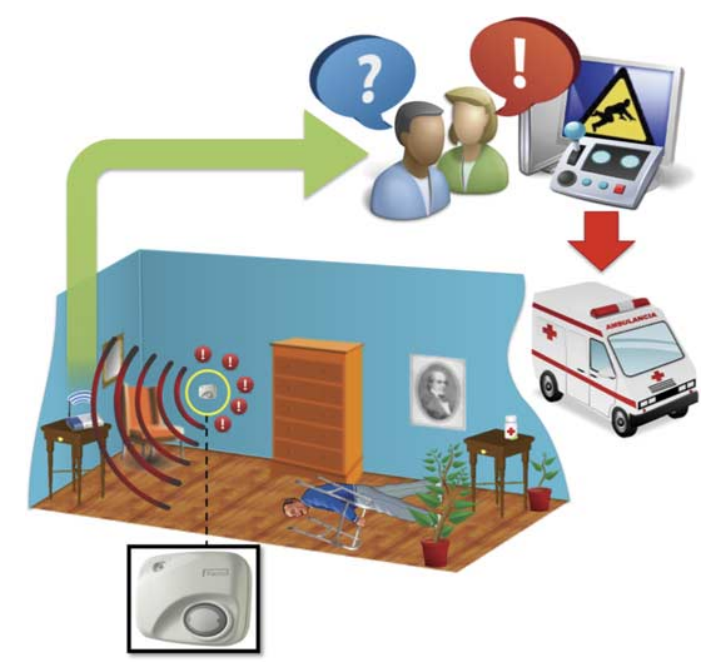

Fig. 11. Scenario 3: Daily activity monitoring by an informal caregiver and possible warning messages.

tivities performed by the person inside the house, e.g., monitoring of ADL activities in the different rooms of the house. Such information can be then presented to the informal caregiver as a further evidence of particular situations that may occur in the house.

The relevant sensors and parameters are mainly environmental sensors and parameters. The principal requirements involved in the scenario are related to detect risky situations, in particular detecting falls, and to provide warning notices to secondary users, to monitor the activities performed in different home places and providing periodic reporting to secondary users.

In this scenario, the main focus is on exploitation of data generated by sensors to check for risky situations (IP 6) as well as generate alarms for informal caregivers (IP 3). Yet another scenario in which data policy and privacy issues management are to be considered (IP 1).

\section{Limitations of the study}

This work presents a systematic analysis on users' needs and preferences on a complex assistive system aimed to support both the elderly live independently in their own homes and their carers. Nevertheless we recognize some limitations of the study which open the path to further investigations.

One of the limit is that our results focus on the Italian situation, while a cross-cultural investigation with a comparable sample in terms of participants would have ensured a wider impact and also the emergence of possible differences among populations.

Additionally the present investigation focused mostly on users' needs in terms of services to be delivered through the system, while less attention has been paid on aspects such as the impact and preference on single components to be deployed in the user's environment. For instance, we relied on the idea of using a telepresence robotics platform as a means to support communication related service but this work did not report the investigation on users' feelings with respect to the robotic platform, its appearance and users' preference on interaction style. Collecting information, especially from the seniors' point of view, about their impression on the robot could have provided additional advice on possible models of interaction to be implemented during the developmental phase. Nevertheless, it is also true that, after the developmental stage, a long-term evaluation phase was foreseen by deploying the whole system into real houses for long-term testing and iterative refinement and this makes plausible to hypothesise that this specific limitation will be faced during the long-term assessment.

Overall the testing phase in real houses will allow users to experience the produced technology, thus complementing the analysis on their feelings and opinion on this kind of assistive technology.

\section{Conclusions}

This paper describes the systematic work devoted to the elicitation and validation of users expectations of AAL intelligent services. Only few evidences and works systematically analyzed users' requirements of such systems. Queirós and colleagues [37] highlighted in their review a deep lack in AAL research with respect to a deep and intense user perspective investigation. More specifically, it has been pointed out that there is a considerable attention focused on how technology can be used in the AAL contexts instead of looking at the users' needs and proposing ways to address them. The focus is still on technology rather than on the person. In this respect, this paper reports on our efforts put on the user requirements elicitations highlighting the role of the different actors involved (both primary and secondary users) and eliciting the level of importance and priority they attribute to different services. The work is intended as a validation of users needs and a way to highlight the specific areas of interest for AAL solutions development. Results show 
that while there are some concerns about privacy, especially by elderly people, have been raised, users still appreciated the potentiality of AAL solutions in supporting independent living and improving QoL. In fact, it also was discovered that these concerns can be overcome when people are aware of what is happening and receive useful feedback/service. In other words, the importance to make users to understand the meaning and the value of what the system can do emerged clearly, highlighting also that a service that is considered useful is also considered more acceptable.

The main critical areas of intervention have been identified and validated by using a combination of qualitative and quantitative research methods. A list of priorities have been derived for each service. The obtained results have then been used to derive a list or priority associated to services and these provide advice for system developers, pointing out the importance of building intelligent technology that can be adapted and personalized according to the varying users requirements. The combination of these requirements can lead to systems that better respond to the individual needs of supporting the elderly live independently in their own homes.

\section{Acknowledgements}

Authors work has been partially funded by the EU FP7 under the GIRAFFPLUS project (contract \#288173). Authors would like to thank all members of the project consortium. Thanks to the journal reviewers for several constructive comments. In memoriam of Silvia Coradeschi for her lead and dedication in the project.

\section{References}

[1] http://www.match.ac.uk/

[2] M.S. Allen, A. Foster and K. Berg, Receiving help at home: The interplay of human and technological assistance, The Journal of Gerontology 56 (2001), 374-382. doi:10.1093/geronb/ 56.6.S374.

[3] B.L. Beattie, N. Whitelaw, M. Mettler and D. Turner, A vision for older adults and health promotion, American Journal of Health Promotion 18(2) (2003), 200-204. doi:10.4278/08901171-18.2.200.

[4] A. Bergland and I. Narum, Quality of life demands comprehension and further exploration, Journal of Aging \& Health 19(1) (2007), 39-61. doi:10.1177/0898264306296766.
[5] D. Bonino and F. Corno, What would you ask to your home if it were intelligent? Exploring user expectations about nextgeneration homes, Journal of Ambient Intelligence and Smart Environments 3 (2011), 111-126.

[6] A. Bowling, D. Bannister, S. Sutton, O. Evans and J. Windsor, A multidimensional model of QoL in older age, Ageing and Mental Health 6 (2002), 355-371. doi:10.1080/ 1360786021000006983.

[7] S. Brownsell, S. Blackburn and M. Hawley, User requirements for an ICT-based system to provide care, support and information access for older people in the community, Journal of Assistive Technologies 6(1) (2012), 5-23. doi:10.1108/ 17549451211214328.

[8] A. Cesta, S. Coradeschi, C. Cortellessa, J. Gonzalez, L. Tiberio and S. Von Rump, Enabling social interaction through embodiment in ExCITE, in: Second Italian Forum on Ambient Assisted Living, Trento, Italy, 2010.

[9] A. Cesta, G. Cortellessa, F. Fracasso, A. Orlandini, C. Fredriksson, M. Lidskog, I. Pettersson, P. Engfeldt, A. Forsberg, B. Östlund, M. Turno and C. Gutierrez, D1.1 user requirements and design principles report, Technical report, GIRAFFPLUS project, 2012.

[10] A. Cesta, G. Cortellessa, R. Rasconi, F. Pecora, M. Scopelliti and L. Tiberio, Monitoring elderly people with the RoboCare domestic environment: Interaction synthesis and user evaluation, Computational Intelligence 27(1) (2011), 60-82. doi:10. 1111/j.1467-8640.2010.00372.x.

[11] N. Charness and K.W. Schaie, Impact of Technology on Successful Aging, Springer, New York, 2003.

[12] S. Coradeschi, A. Cesta, G. Cortellessa, L. Coraci, J. Gonzalez, L. Karlsson, F. Furfari, A. Loutfi, A. Orlandini, F. Palumbo, F. Pecora, S. von Rump, A. Stimec, J. Ullberg and B. Ostlund, GiraffPlus: Combining social interaction and long term monitoring for promoting independent living, in: HSI: 6th International Conference on Human System Interaction, 2013.

[13] K.E. Covinsky, J. Hilton, K. Lindquist and R.A. Dudley, Development and validation of an index to predict activity of daily living dependence in community-dwelling elders, Medical Care 44(2) (2006), 149-157. doi:10.1097/01.mlr.0000196955. 99704.64.

[14] K.E. Covinsky, R. Newcomer, P. Fox, J. Wood, L. Sands, K. Dane and K. Yaffe, Patient and caregiver characteristics associated with depression in caregivers of patients with dementia, Journal of General Internal Medicine 18 (2001), 10061014. doi:10.1111/j.1525-1497.2003.30103.x.

[15] G. Demiris, M.J. Rantz, M.A. Aud, K.D. Marek, H.W. Tyrer, M. Skubic and A.A. Hussam, Older adults' attitudes towards and perceptions of 'smart home' technologies: A pilot study, Medical Informatics and the Internet in Medicine 29 (2004), 87-94. doi:10.1080/14639230410001684387.

[16] S. Doménech, J. Rivero, L. Coll-Planas, F.J. Sainz, A. Reissner and F. Miralles, Involving older people in the design of an innovative technological system promoting active aging: The SAAPHO project, Journal of Accessibility and Design for All 3 (2013), 13-27.

[17] B. Eggen, G. Hollemans and R. van de Sluis, Exploring and enhancing the home experience, Cognition, Technology and Work 5 (2003), 44-54. doi:10.1007/s10111-002-0114-7.

[18] European Commission, The 2012 ageing report - Economic and budgetary projections for the EU-27 member states (2010 2060) Brussels: Joint report prepared by the European Com- 
mission (DG ECFIN) and the Economic Policy Committee (AWG), Technical report, European Economy, 2012.

[19] C.B. Fausset, A.J. Kelly, W.A. Rogers and A.D. Fisk, Challenges to aging in place: Understanding home maintenance difficulties, Journal of Housing for the Elderly 25(2) (2011), 125 141. doi:10.1080/02763893.2011.571105.

[20] M. Gordon, Community care for the elderly: Is it really better?, Canadian Medical Association Journal 148 (1993), 393-396.

[21] D.L. Gray-Miceli, N.E. Strumpf, J. Johnson, M. Draganescu and S.J. Ratcliffe, Psychometric properties of the post-fall index, Clinical Nursing Research 15(3) (2006), 157-176. doi:10. 1177/1054773806288566.

[22] L. Griffith, P. Raina, H. Wu, B. Zhu and L. Stathokostats, Population attributable risk for functional disability associated with chronic conditions in Canadian older adults, Age and Ageing 39 (2010), 738-745. doi:10.1093/ageing/afq105.

[23] S.M. Heidrich, Health promotion in old age, Annual Review of Nursing Research 16 (1998), 173-195.

[24] A. Hein, A. Hein, S. Winkelbach, B. Martens, O. Wilken, M. Eichelberg, J. Spehr, M. Gietzelt, K.H. Wolf, F. Büsching, M. Hülsken-Giesler, M. Meis and P. Okken, Monitoring systems for the support of home care, Informatics for Health and Social Care 35(3-4) (2010), 157-176. doi:10.3109/17538157. 2010.528637.

[25] Y. Hellström and I.R. Hallberg, Perspectives of elderly people receiving home help on health, care and quality of life, Health and Social Care in the Community 9(2) (2001), 61-71. doi:10. 1046/j.1365-2524.2001.00282.x.

[26] P.G. Higgins, Biometric outcome of a geriatric health promotion programme, Journal of Advanced Nursing 13(6) (1988), 710-715. doi:10.1111/j.1365-2648.1988.tb00561.x.

[27] S. Iwarsson, A long-term perspective on person-environment fit and ADL dependence among older Swedish adults, Gerontologist 45 (2005), 327-336. doi:10.1093/geront/45.3.327.

[28] J.J. Keysor, A.M. Jette, W. Coster, J.P. Bettger and S.M. Haley, Association of environmental factors with levels of home and community participation in an adult rehabilitation cohort, Archives of Physical Medicine and Rehabilitation 87(12) (2006), 1566-1575. doi:10.1016/j.apmr.2006.08.347.

[29] B.E.K. Klein, R. Klein, M.D. Knudtson and K.E. Lee, Frailty, morbidity and survival, Archives of Gerontology and Geriatrics 41 (2005), 141-149. doi:10.1016/j.archger.2005.01.002.

[30] K. Marek and M. Rantz, Aging in place: A new model for longterm care, Nursing Administration Quarterly 24(3) (2000), 1-11. doi:10.1097/00006216-200004000-00003.

[31] J.L. Martin, L. Fiorentino, S. Jouldjian, K.R. Josephson and C.A. Alessi, Sleep quality in residents of assisted living facilities: Effect on quality of life, functional status, and depression, Journal of the American Geriatrics Society 58(5) (2010), 829836. doi:10.1111/j.1532-5415.2010.02815.x.

[32] S. Martin, J.C. Augusto, P. McCullagh, W. Carswell, H. Zheng, H. Wang, J. Wallace and M. Mulvenna, Participatory research to design a novel Telehealth system to support the nighttime needs of people with dementia: NOCTURNAL, International Journal of Environmental Research and Public Health 10 (2013), 6764-6782. doi:10.3390/ijerph10126764.
[33] J.A. Mayer, A. Jermanovich, B.L. Wright, J.P. Elder, J.A. Drew and S.J. Williams, Changes in health behaviors of older adults, Preventive Medicine 23(2) (1994), 127-133. doi:10. 1006/pmed.1994.1018.

[34] A.D. Naik, M.E. Kunik, K.R. Cassidy, J. Nair and J. Coverdale, Assessing safe and independent living in vulnerable older adults: Perspectives of professionals who conduct home assessments, Journal of the American Board of Family Medicine 23(5) (2010), 614-621. doi:10.3122/jabfm.2010.05.090065.

[35] A. Odlund Olin, A. Koochek, O. Ljungqvist and T. Cederholm, Nutritional status, well-being and functional ability in frail elderly service flat residents, European Journal of Clinical $\mathrm{Nu}$ trition. 59 (2005), 263-270. doi:10.1038/sj.ejcn.1602067.

[36] R.H. Osborne, G. Hawthorne, E.A. Lew and L.C. Gray, Quality of life assessment in the community-dwelling elderly: Validation of the assessment instrument and comparison with SF 36, Journal of Clinical Epidemiology 56(2) (2003), 138-147. doi:10.1016/S0895-4356(02)00601-7.

[37] A. Queirós, A. Silva, J. Alvarelhão, N. Pacheco Rocha and A. Teixeira, Usability, accessibility and ambient-assisted living: A systematic literature review, Universal Access in the Information Society 14 (2015), 27-66. doi:10.1007/s10209-0130328-x.

[38] D. Raphael, I. Brown, R. Renwick, M. Cava, N. Weir and $\mathrm{K}$. Healthcote, The quality of life of seniors living in the community: A conceptualization with implications for public health practice, Canadian Journal of Public Health 86 (1995), 228-233.

[39] A.C. Scheffer, M.J. Schuurmans, N. van Dijk, T. van der Hooft and S.E. de Rooij, Fear of falling: Measurement strategy, prevalence, risk factors and consequences among older persons, Age and Ageing 37 (2008), 19-24. doi:10.1093/ageing/ afm169.

[40] L. Schelisch and A. Spellerberg, Two steps forward and one step back? On the acceptance and use of AAL technology in household, in: Ambient Assisted Living, Advanced Technologies and Societal Changes, R. Wichert and B. Eberhardt, eds, Springer, Berlin, 2012, pp. 185-196.

[41] D.W. Stewart, P.N. Shamdasani and D.W. Rook, Focus Groups: Theory and Practice, Sage Publications, Thousand Oaks, CA, 2007. doi:10.4135/9781412991841.

[42] The Whoqol Group, The World Health Organization Quality of Life Assessment (WHOQOL): Development and general psychometric properties, Psychological Medicine 28(3) (1998), 551-558. doi:10.1017/S0033291798006667.

[43] K. Wild, L. Boise, J. Lundell and A. Foucek, Unobtrusive inhome monitoring of cognitive and physical health: Reactions and perceptions of older adults, Journal of Applied Gerontology 27 (2008), 101-200.

[44] World Health Organization, International Classification of Functioning, Disability and Health (ICF), World Health Organization, Geneva, 2001

[45] T. Yamanaka, E. Takasugi, N. Hotta, Y. Kubo and K. Otsuka, Daily living functions of the elderly requiring home visits: A study at a comprehensive assessment clinic for the elderly, Geriatrics \& Gerontology International 7(4) (2007), 388-392. doi:10.1111/j.1447-0594.2007.00429.x. 